\title{
ADSORÇÃO DE XANTATOS SOBRE PIRITA
}

Fábio Garcia Penha, Vivian Cristina Spier e Nito Angelo Debacher

Departamento de Química, Universidade Federal de Santa Catarina, CP 476, 88040-900 Florianópolis - SC

Recebido em 18/7/00; aceito em 22/1/01

\begin{abstract}
ADSORPTION OF XANTHATE ON PYRITE. This paper presents a study of adsorption of xanthate with alkyl chain of two $\left(\mathrm{C}_{2} \mathrm{XK}\right)$, four $\left(\mathrm{C}_{4} \mathrm{XK}\right)$ and eight $\left(\mathrm{C}_{8} \mathrm{XK}\right)$ atoms of carbon, on pyrite from Santa Catarina, Brazil. The results showed that pyrite surface changes from hydrophilic to hydrophobic when $\mathrm{xanthate}$ is adsorbed increasing the contact angle to $35^{\circ}$ for $\mathrm{C}_{2} \mathrm{XK}$, and to $90^{\circ}$ for $\mathrm{C}_{4} \mathrm{XK}$ and $\mathrm{C}_{8} \mathrm{XK}$. The rate of flotation of pyrite particles after adsorption increases with the increase of the number of carbon atoms in the alkyl chain in agreement with the results of contact angle measurements.
\end{abstract}

Keywords: pyrite; adsorption; contact angle.

\section{INTRODUÇÃO}

A adsorção é um processo de concentração de uma espécie química entre duas fases diminuindo a energia livre superficial, liberando ou absorvendo uma quantidade definida de ca$\operatorname{lor}^{1-2}$. A adsorção pode ser física ou química e o processo é governado por um grande número de interações na região interfacial envolvendo muitas variáveis tais como a solubilidade e a carga superficial do sólido, o $\mathrm{pH}$, a temperatura da solução e a própria estrutura da espécie química que se adsorve.

Através da adsorção de determinadas moléculas na interface adsorvente adsorbato é possível controlar vários processos importantes como a separação do carvão mineral de impurezas como a pirita por flotação ${ }^{3-5}$. Os adsorbatos comumente usados na flotação de pirita são compostos organossulfurados, tais como os xantatos, com capacidade de tornar superfícies hidrofílicas como a pirita em hidrofóbicas ${ }^{6-10}$.

A flotação depende basicamente da criação de um interface de equilíbrio sólido/líquido/gás, com um ângulo de contato maior que zero grau, que depende da hidrofobicidade da superfície do sólido $^{11}$. A hidrofobicidade do sólido pode ser medida pelo ângulo de contato, $(\theta)$, formado entre as três fases em equilíbrio ${ }^{1,12-15}$. Quando o sólido é totalmente hidrofóbico não há afinidade da água em sua superfície e o sólido não é molhável e o ângulo de contato se aproxima de $180^{\circ}$. Para sólidos hidrofílicos ou seja molháveis o ângulo de contato se aproxima de zero grau.

Entre os vários métodos experimentais para a medida do ângulo de contato ${ }^{16-21}$ um dos mais adequados é o método da captura da bolha ${ }^{22}$. Este método consiste em gerar uma bolha de gás em um meio líquido na presença de um sólido possibilitando desta forma manter em equilíbrio a pressão de vapor na fase gasosa. Para efetuar as medidas as três fases são colocadas em contato e a leitura do ângulo é feita em relação a fase líquida. Neste caso o método permite manter o equilíbrio entre as três fases durante os experimentos evitando a perda de material por evaporação.

Neste trabalho serão apresentados resultados de adsorção, ângulo de contato e flotação para o sistema xantato/pirita com o $\mathrm{pH}$ e a temperatura constantes.

\section{PARTE EXPERIMENTAL}

\section{Materiais e métodos}

Os xantatos foram sintetizados conforme descrito na literatu$\mathrm{ra}^{23}$ pela adição de hidróxido de potássio em etanol, butanol e octanol respectivamente. Em seguida adicionou-se lentamente e sob agitação dissulfeto de carbono. Os produtos formados foram recristalizados em etanol a frio.

Os xantatos sintetizados foram: etil xantato de potássio $\left(\mathrm{C}_{2} \mathrm{XK}\right)$, butil xantato de potássio $\left(\mathrm{C}_{4} \mathrm{XK}\right)$ e octil xantato de potássio $\left(\mathrm{C}_{8} \mathrm{XK}\right)$. Todos apresentaram absorbância máxima no UV-Vis em comprimento de onda de $301 \mathrm{~nm}$, e absortividade molar em água de $16.000 \mathrm{~L} \mathrm{~mol}^{-1} \mathrm{~cm}^{-1}$ para o $\mathrm{C}_{2}, 15.325 \mathrm{~L} \mathrm{~mol}^{-1} \mathrm{~cm}^{-1}$ para o $\mathrm{C}_{4}$ e $14.900 \mathrm{~L} \mathrm{~mol}^{-1} \mathrm{~cm}^{-1}$ para o $\mathrm{C}_{8}$. $\mathrm{O} \mathrm{C}_{8}$ foi o único que apresentou concentração micelar crítica (CMC) de $1,90 \times 10^{-2}$. mol L ${ }^{-1}$.

A pirita foi coletada na Mina Esperança, da Carbonífera Metropolitana em Siderópolis, SC. A amostra foi pulverizada e classificada em um conjunto de peneiras vibratórias. A granulometria utilizada na adsorção foi na faixa de 0,210 a $0,297 \mathrm{~mm}$ de diâmetro e na flotação foi de 0,105 a $0,125 \mathrm{~mm}$ de diâmetro. A área superficial específica, $\mathrm{A}_{\text {esp., }}$, foi calculada por adsorção de $\mathrm{N}_{2}$ e os dados ajustados de acordo com a equação de BET. O valor encontrado foi de $0,246 \mathrm{~m}^{2} \mathrm{~g}^{-1}$ para as partículas na faixa de 0,210 a $0,297 \mathrm{~mm}$ e $0,516 \mathrm{~m}^{2} \mathrm{~g}^{-1}$ para as partículas de 0,105 a $0,125 \mathrm{~mm}$. A microanálise por energia dispersiva, indicou a presença, além do ferro e do enxofre, de traços de carbono, silício e alumínio, provenientes de aluminossilicatos. Para as medidas de ângulo de contato a pirita foi cortada em placas de $1,0 \times 2,0 \mathrm{~cm}$ e polida usando lixa d'água de 300 mesh antes de cada medida.

As análises de infravermelho da pirita adsorvida com $\mathrm{C}_{2} \mathrm{XK}$, $\mathrm{C}_{4} \mathrm{XK}$ e $\mathrm{C}_{8} \mathrm{XK}$ foram feitas utilizando $5 \%$ da amostra em pastilhas de $\mathrm{KBr}$ num aparelho Perkin Elmer 16PC com transformada de Fourier.

Os experimentos de adsorção foram realizados em um reator de vidro, (Figura 1) sob um fluxo de oxigênio ${ }^{24}$, em $\mathrm{pH}=$ 8,00 , com temperatura e agitação controlados. A quantidade adsorvida foi acompanhada por UV, pelo desaparecimento dos xantatos em $301 \mathrm{~nm}$ usando-se $1,0 \mathrm{~g}$ de pirita e $50 \mathrm{~mL}$ da solução aquosa dos respectivos xantatos de concentração conhecida. As isotermas foram construídas adicionando-se quantidades conhecidas dos xantatos até a saturação da superfície da pirita. Para efeito experimental foi considerado como concentração inicial o valor da absorbância antes do inicio da adsorção e a concentração de equilíbrio quando não havia mudanças na absorbância.

A flotação foi realizada após a adsorção dos xantatos, num tubo de Hallimond modificado ${ }^{25}$, utilizando gás nitrogênio para produzir as bolhas em $\mathrm{pH}=8,00$, com um fluxo de $0,5 \mathrm{~L} /$ minuto, temperatura de $25^{\circ} \mathrm{C}$, tamanho das partículas de 0,105 a $0,125 \mathrm{~mm}$.

As medidas do ângulo de contato foram feitas pelo método da captura da bolha usando o equipamento mostrado na Figura 
2, a $25^{\circ} \mathrm{C}$ e $\mathrm{pH}=8,00$. Pela análise do perfil da bolha, o equipamento permite fazer medidas de ângulos maiores de $5^{\circ}$ \pm 2 , porém quanto maior o ângulo maior a precisão nas medidas. Foram usadas bolhas de raio médio de $1,0 \mathrm{~mm}$ e as medidas do ângulo foram feitas com auxílio de um micro computador usando o programa para análises e processamento de imagens da "UTHSCSA ImageTool". Antes de cada medida do ângulo, para uma dada concentração, foi feita a adsorção do xantato na pirita até atingir o equilíbrio adsorção/dessorção. Para as medidas do ângulo as três fases sólido/líquido/gás foram colocadas em contato. Durante os experimentos o ângulo de contato foi monitorado fazendo-se leituras até atingir valores constantes. Estas medidas foram consideradas ângulo de contato de equilíbrio.

As Figuras 3a e 3b mostram um exemplo típico do ângulo de contato para a pirita sem xantato adsorvido (Figura $3 \mathrm{a}, \theta=0^{\circ}$ ) e após a adsorção de $\mathrm{C}_{8} \mathrm{XK}$ (Figura $3 \mathrm{~b}, \theta=90^{\circ}$ ). Foi considerado ângulo zero quando não havia adesão da bolha na superfície da pirita mesmo após um longo período em contato.

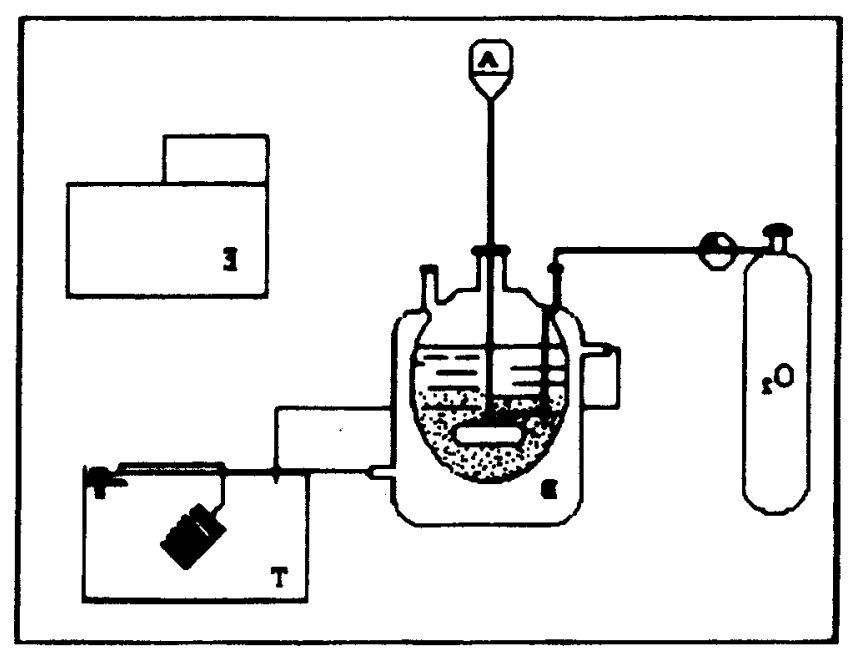

Figura 1. Sistema utilizado nos experimentos de adsorção; $\mathrm{O}_{2}$ cilindro de oxigênio, $B$ - balão termostatizado; $T$ - termostato, $A$ - agitador mecânico e E - espectrofotômetro UV-vis.

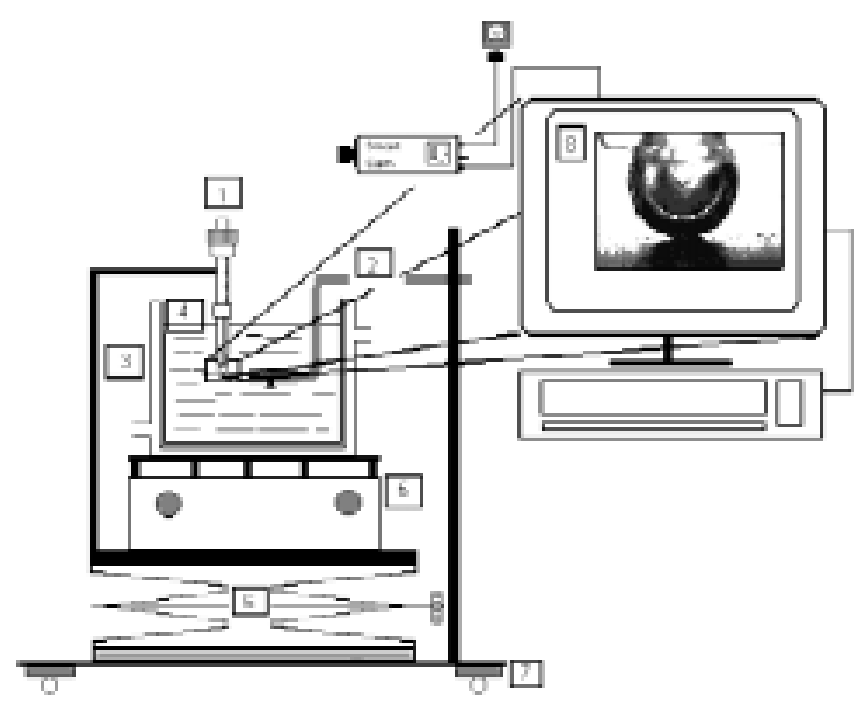

Figura 2. Aparelho de medidas de ângulo de contato: 1 micrômetro Gilmont de 0,2 mL; 2 - suporte da amostra sólida; 3 - suporte da cubeta; 4 - cubeta de vidro ótico; 5 - agitador magnético; 6 - regulador de altura; 7 - mesa antivibração; 8 - imagem capturada; 9 - câmara de vídeo.

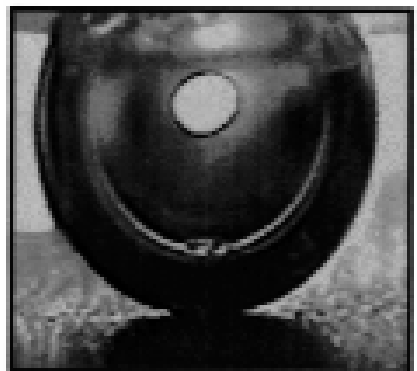

(a)

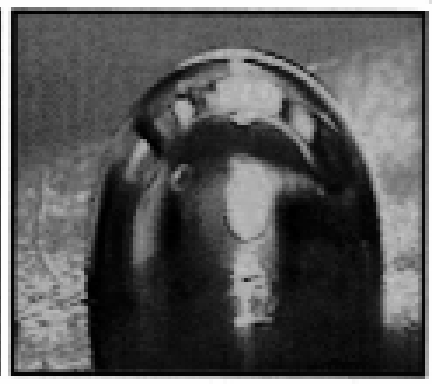

(b)
Figura 3. (a) - Sistema, pirita-água-ar, ângulo de $0^{o} ;(b)-$ sistema, pirita- $C_{8} X K$-ar, ângulo de contato de $90^{\circ}$. Concentração inicial de $\left[C_{8} X K\right]=1 \times 10^{-4} \mathrm{~mol} \mathrm{~L} L^{-1}, p H=8,00$ e $T=25^{\circ} \mathrm{C}$.

\section{RESULTADOS E DISCUSSÃO}

\section{Isotermas de Adsorção, Ângulo de Contato e Flotação}

A Figura 4 mostra as isotermas de adsorção para o $\mathrm{C}_{2} \mathrm{XK}$, o $\mathrm{C}_{4} \mathrm{XK}$ e o $\mathrm{C}_{8} \mathrm{XK}$. A quantidade de xantato adsorvido em mol por grama de pirita, em função da concentração de equilíbrio diminui com o aumento do tamanho da cadeia carbônica. Para o $\mathrm{C}_{2} \mathrm{XK}$ a saturação da superfície foi atingida com uma quantidade de aproximadamente $17,0 \times 10^{-5} \mathrm{molg}^{-1}$, para o $\mathrm{C}_{4} \mathrm{XK}$ de $9,0 \times 10^{-5} \mathrm{molg}^{-1}$ e para o $\mathrm{C}_{8} \mathrm{XK}$ de $3,0 \times 10^{-5} \mathrm{molg}^{-1}$. Estes resultados mostram que com o aumento do número de átomos de carbono na molécula, menor é a quantidade de moléculas adsorvidas na superfície. Resultados similares de adsorção com $\mathrm{C}_{2} \mathrm{XK}, \mathrm{C}_{5} \mathrm{XK}$ e $\mathrm{C}_{8} \mathrm{XK}$ sobre galena mostrados por Fuerstenau et al. ${ }^{26}$, também apresentaram uma diminuição na quantidade de xantato adsorvida com o aumento da cadeia carbônica das moléculas.

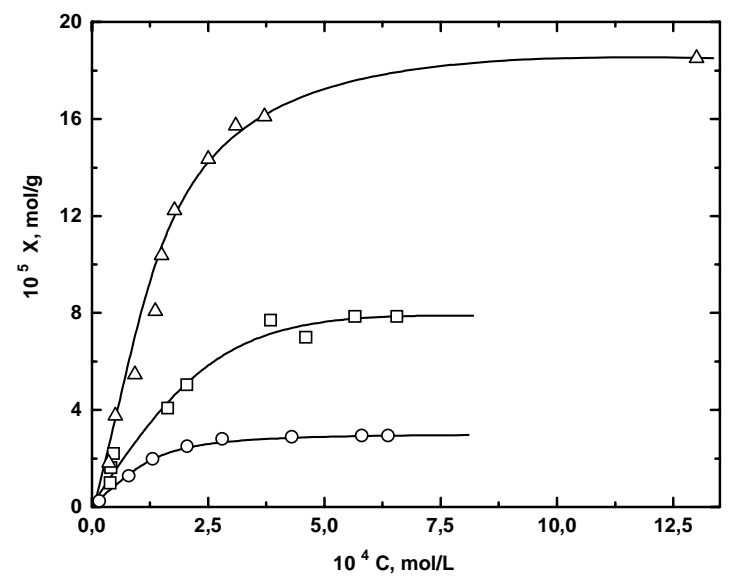

Figura 4. Isotermas de adsorção, $T=25^{\circ} \mathrm{C}, p H=8,00, \Delta C_{2} X K$, $\square C_{4} X K$ e $O C_{8} X K$.

A Figura 5 mostra os espectros de FTIR para os três xantatos estudados após a adsorção sobre a pirita. $\mathrm{O}$ espectro (a) mostra a pirita pura antes da adsorção. As bandas na região de $900-1100 \mathrm{~cm}^{-1}$ são devido à produtos de oxidação da pirita, como por exemplo $\mathrm{FeSO}_{4} \cdot \mathrm{xH}_{2} \mathrm{O}$. Os espectros $(b),(c)$ e $(d)$ mostram a pirita saturada com $\mathrm{C}_{2} \mathrm{XK}$, com $\mathrm{C}_{4} \mathrm{XK}$ e $\operatorname{com} \mathrm{C}_{8} \mathrm{XK}$, respectivamente. Os espectros são semelhantes, e a banda na região de $1026 \mathrm{~cm}^{-1}$ é devido ao estiramento da ligação $\mathrm{C}=\mathrm{S}$. A banda na região de $1260 \mathrm{~cm}^{-1}$ é devido ao estiramento da ligação $\mathrm{C}-\mathrm{O}-\mathrm{C}$, característico somente do dixantogênio, produto da dimerização dos xantatos. As bandas na região de 1400 $\mathrm{cm}^{-1}$ e $3000 \mathrm{~cm}^{-1}$ são devidas a torção e ao estiramento da ligação $\mathrm{C}-\mathrm{H}$ da cadeia carbônica respectivamente. 


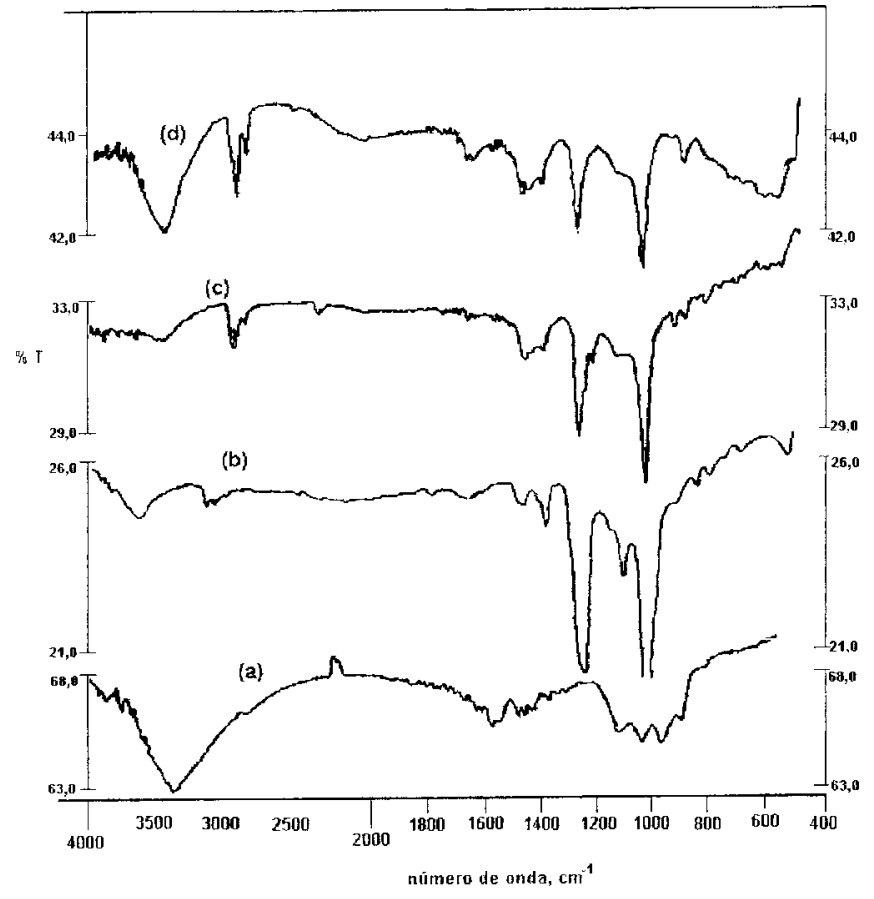

Figura 5. Espectros de FTIR da pirita: (a) - sem xantato adsorvido; (b) - saturada com $C_{2} X K$; (c) - saturada com $C_{4} X K$ $e(d)$ - saturada com $C_{8} X K$.

Trabalhos anteriores ${ }^{6,10,24}$ mostraram que, no caso do $\mathrm{C}_{2} \mathrm{XK}$, a espécie que se adsorve na superfície da pirita é o dixantogênio e é dependente do nível de oxidação da superfície da pirita e da presença de oxigênio na solução. Fuerstenau et al. ${ }^{27}$, identificou através de análise de infra-vermelho a espécie adsorvida para o sistema $\mathrm{C}_{10} \mathrm{XK} /$ pirita apenas o decil dixantogênio. Buckley ${ }^{28}$, através de estudos usando a espectroscopia fotoeletrônica de raioX (XPS), também caracterizou os xantatos adsorvidos em minerais sulfurados como sendo o dixantogênio correspondente.

A Figura 6 mostra a dependência do ângulo de contato de equilíbrio para os xantatos nas diferentes concentrações iniciais. Para o $\mathrm{C}_{2} \mathrm{XK}$ o ângulo de contato máximo foi de $35^{\circ}$ e para o $\mathrm{C}_{4} \mathrm{XK}$ e o $\mathrm{C}_{8} \mathrm{XK}$ foi de $90^{\circ}$. Os dados mostram que em concentrações baixas de xantato o ângulo de contato aumenta rapidamente passando de zero grau para pirita em água pura para $20^{\circ}$ na concentração inicial de $1,0 \times 10^{-4} \mathrm{~mol} \mathrm{~L}^{-1} \mathrm{de}$ $\mathrm{C}_{2} \mathrm{XK}$, e $33^{\circ}$ para o $\mathrm{C}_{4} \mathrm{XK}$ e o $\mathrm{C}_{8} \mathrm{XK}$ nas concentrações iniciais de $5,6 \times 10^{-6} \mathrm{~mol} \mathrm{~L}^{-1}$ e $1,0 \times 10^{-6} \mathrm{~mol} \mathrm{~L}^{-1}$ respectivamente. Isto indica que pequenas quantidades de xantato adsorvido é suficiente para tornar a pirita parcialmente hidrofóbica ou seja com ângulo de contato maior que zero grau.

Resultados similares de ângulo de contato foram obtidos no trabalho de Gardner et al. ${ }^{29}$, usando os xantatos, $\mathrm{C}_{1} \mathrm{XK}$, $\mathrm{C}_{2} \mathrm{XK}$ e $\mathrm{C}_{4} \mathrm{XK}$. Os ângulos de contato foram medidos diretamente sobre bastonetes de pirita (eletrodo) após a aplicação de uma diferença de potencial usando uma solução de xantato de $1000 \mathrm{ppm}$. Eles observaram que em potenciais baixos a pirita era hidrofílica com ângulo de contato de zero grau e aumentando o potencial até $0,3 \mathrm{~V}$., havia a formação de dixantogênio na superfície da pirita por oxidação eletroquímica. O ângulo de contato máximo obtido nestas condições foi de $90^{\circ}$ para o $\mathrm{C}_{4} \mathrm{XK}$.

Os dados de adsorção mostram que quanto maior a cadeia carbônica da molécula dos xantatos menor é a quantidade adsorvida por grama de pirita necessária para atingir o valor máximo de adsorção na isoterma da Figura 4. No entanto os dados de ângulo de contato (Figura 5) mostram que com o aumento da cadeia carbônica da molécula dos xantatos menor é a quantidade necessária para tornar a superfície da pirita mais hidrofóbica ou seja o ângulo de contato de equilíbrio passa de $30^{\circ}$ para $\mathrm{C}_{2} \mathrm{XK}$, a $90^{\circ}$ para o $\mathrm{C}_{4} \mathrm{XK}$ e $\mathrm{C}_{8} \mathrm{XK}$ no máximo das curvas das isotermas de adsorção da Figura 4.

Fuerstenau et $a l .{ }^{26}$, calcularam, para vários xantatos de cadeias carbônicas diferentes, o número de moléculas necessárias para cobrir com uma monocamada toda a área superficial das partículas de galena $(\mathrm{PbS})$. Eles assumiram que a cada cela unitária dois átomos de chumbo estão disponíveis para interação e que apenas uma molécula do xantato se adsorve sobre um átomo de chumbo. $\mathrm{O}$ que eles observaram foi que, com o aumento da cadeia carbônica, menos moléculas se adsorveram na superfície, chegando a ser inferior a uma monocamada. Eles atribuíram este fato ao efeito estérico entre as moléculas.

A Figura 7 mostra que a porcentagem de pirita recuperada na flotação aumenta linearmente com o tamanho da cadeia carbônica dos xantatos. Os experimentos realizados numa concentração inicial dos xantatos de $2,0 \times 10^{-4} \mathrm{~mol} \mathrm{~L}^{-1}$ e um tempo de flotação de 1000 segundos mostram que a recuperação foi de $90 \%$ para o $\mathrm{C}_{8} \mathrm{XK}$, de $57 \%$ para $\mathrm{C}_{4} \mathrm{XK}$, de $37 \%$ para o $\mathrm{C}_{2} \mathrm{XK}$ e $20 \%$ na ausência de xantato. $\mathrm{O}$ coeficiente angular obtido da reta foi de 8,5. Este valor indica que o aumento de 1 átomo de carbono na cadeia do xantato aumenta em 8,5 vezes a porcentagem de pirita recuperada no mesmo tempo de flotação. $\mathrm{O}$ ângulo de contato nesta concentração para o $\mathrm{C}_{8} \mathrm{XK}$ é de $90^{\circ}$, para o $\mathrm{C}_{4} \mathrm{XK}$ é de $75^{\circ}$, para o $\mathrm{C}_{2} \mathrm{XK}$ é de $30^{\circ}$ e para a pirita sem xantato adsorvido é zero grau.

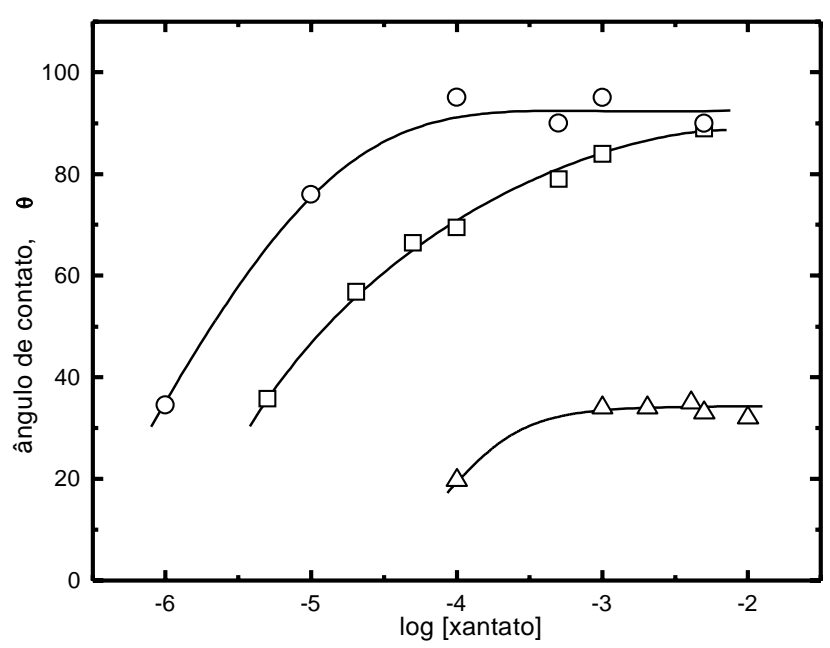

Figura 6. Ângulo de contato de equilíbrio para o sistema piritaxantato-ar; $T=25^{\circ} \mathrm{C}, \mathrm{pH}=8,00, \Delta C_{2} X K, \square C_{4} X K$ e O $C_{8} X K$.

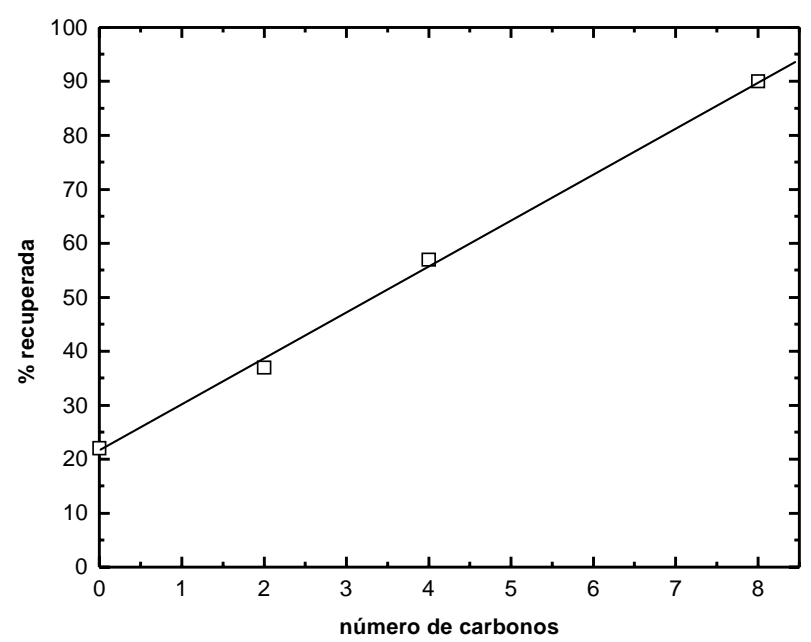

Figura 7. Efeito do tamanho da cadeia carbônica dos xantatos na porcentagem de pirita recuperada. Tempo de flotação 1000 segundos, concentração dos xantatos $=2,0 \times 10^{-4} \mathrm{~mol} \mathrm{~L}^{-1}, \mathrm{pH}$

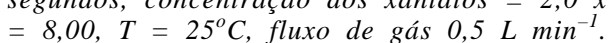


A efetividade do processo de flotação depende em grande parte do balanço de energias interfacias, expressa pelo trabalho de adesão. Com o aumento da cadeia carbônica da molécula adsorvida, aumenta a hidrofobicidade da superfície da pirita e com isto aumenta o ângulo de contato favorecendo a adesão partícula-bolha.

A flotação também ocorre na ausência de xantato, o que sugere que a flotação neste caso depende apenas do fluxo de gás e do tamanho das partículas. Quanto menor o tamanho das partículas ou quanto maior o fluxo de gás de arraste, maior é a porcentagem de partículas recuperadas ${ }^{25}$ nestas condições. Neste caso a flotação independe do ângulo de contato formado entre as três fases sólido/líquido/gás, dependendo apenas da turbulência causada no sistema pelo fluxo de gás e da geometria do tubo de Hallimond usado no experimento ${ }^{25,30}$.

A concentração inicial mínima dos xantatos para que a recuperação da pirita seja total após 100 segundos de flotação variou com o tamanho da cadeia carbonica do xantato. A Figura 8 mostra que para o $\mathrm{C}_{8} \mathrm{XK}$ a recuperação é total na concentração de $6,0 \times 10^{-4} \mathrm{~mol} \mathrm{~L}^{-1}$ (eqüivale a $1,2 \times 10^{-5} \mathrm{molg}^{-1}$ de xantato adsorvido), para o $\mathrm{C}_{4} \mathrm{XK} 1,0 \times 10^{-3} \mathrm{~mol} \mathrm{~L}^{-1}$ (eqüivale a $2,9 \times$ $10^{-5} \mathrm{molg}^{-1}$ de xantato adsorvido) e para o $\mathrm{C}_{2} \mathrm{XK} 3,010^{-3}$ mol L ${ }^{-1}$ (equiivale a $5,8 \times 10^{-5} \mathrm{molg}^{-1}$ de xantato adsorvido).

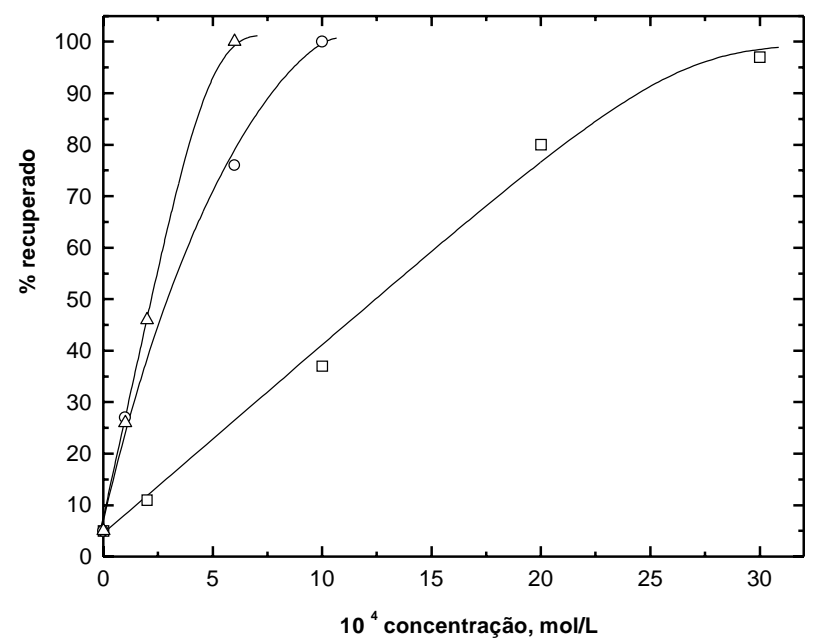

Figura 8. Porcentagem de pirita recuperada em função da concentração inicial dos xantatos. Tempo de flotação 100 segundos, fluxo de gás $0,5 \mathrm{~L} \mathrm{~min}^{-1}, \mathrm{~T}=25^{\circ} \mathrm{C}, \mathrm{pH}=8,0, \Delta C_{8} \mathrm{XK}, \mathrm{O}$ $\mathrm{C}_{4} \mathrm{XK}$ e $\mathrm{C}_{2} \mathrm{XK}$.

\section{CONCLUSÃO}

A hidrofobicidade da pirita aumentou com o aumento do número de átomos de carbono na cadeia dos xantatos. Este fato pode ser verificado pelo aumento do ângulo de contato com o aumento do número de carbonos na cadeia dos xantatos (Figura 6).

A diminuição do número de moléculas necessárias para a saturação da superfície com o aumento da cadeia carbônica (Figura 4) está relacionado com o aumento da área ocupada por molécula de dixantogênio adsorvida. Com isto menos moléculas são necessárias para o total recobrimento da superfície. A orientação das cadeias estão determinando a quantidade de xantato adsorvido e a eficiência na hidrofobização da pirita. Portanto os resultados de adsorção e ângulo de contato indicam que as cadeias não estão perfeitamente orientadas perpendicularmente à superfície.

$\mathrm{O}$ aumento da cadeia carbônica dos xantatos diminui o tempo de residência das partículas no tubo de Hallimond durante a flotação. Em determinadas condições de fluxo e tamanho de partículas a flotação é um processo que depende basicamente da estabilidade da partícula aderida à bolha ${ }^{25,30}$. Neste caso, quanto mais hidrofóbica for a superfície mais favorável se tornará este processo e consequentemente mais rápida será a recuperação do mineral.

Os resultados da isoterma de adsorção mostram que a quantidade de xantato necessária para que a flotação das partículas seja de $100 \%$ nas condições do experimento é bem menor do que a quantidade necessária para atingir o máximo de adsorção. Isto pode ser explicado se considerarmos que o processo de adsorção se inicia em pequenos pontos da superfície onde os sítios ativos apresentam maior atividade fazendo com que uma concentração maior de moléculas se adsorvam nestes sítios. Desta forma a aderência da partícula à bolha de gás formada no sistema pode ocorrer mesmo que parte da partícula ainda não seja totalmente hidrofóbica e o ângulo de contato seja menor que $90^{\circ}$. Estes resultados permitem concluir que a superfície não precisa estar inteiramente coberta com uma monocamada adsorvida ou seja completamente hidrofóbica para que a recuperação das partículas seja de $100 \%$ nas condições em que os experimentos foram realizados e que as características superficiais das partículas como distribuição de cargas, composição química e forma física podem favorecer o processo tanto na adsorção bem como na flotação.

\section{REFERÊNCIAS}

1. Adamson, A . W.; Physical Chemistry of Surfaces; A Wiley Interscience Publication, fifth edition, California, 1990.

2. Atkins, P. W.; Físico-Química, volume 3, sexta edição, LTC-Livros Técnicos e Científicos, Rio de Janeiro, 1999.

3. Jiang, C. L.; Wang, X. H.; Parekh, B. K.; Leonard, J. W.; Colloids Surf. A 1998, 133, 51.

4. Kydros, K.; Matis, K.; Stalidis, G.; J. Colloid Interface Sci. 1993, 155, 409.

5. Fornasiero, D.; Eijt. J; Ralston, J.; Colloids Surf. 1992, $62,63$.

6. Montalti, M.; Fornasiero, D.; Ralston, J.; J. Colloid Interface Sci. 1991, 143, 440.

7. Fornasiero, D.; Ralston, J.; J. Colloid Interface Sci. 1992, $151,225$.

8. Valli, M.; Persson, P.; Persson, I.; Colloids Surf. 1991, 59, 293.

9. Valli, M.; Persson, I.; Colloids Surf. A 1994, 83, 199.

10. Cases, J. M. ; De Donato, P.; Kongolo, M.; Michot, L.; Colloids Surf. 1989, 36, 323.

11. Leja, J.; Surface Chemistry of Froth Flotation, Plenum Press, New York, 1982.

12. Li, D.; Colloids Surf. A 1996, 116, 1.

13. Marmur, A ; Colloids Surf. A 1996, 116, 55.

14. Drelich, J.; Miller, J. D.; J. Colloid Interface Sci. 1994, 164, 252.

15. Hiemenz, P. C. - Principles of Colloid and Surface Chemistry; Marcel Dekker, second edition, New York, 1987.

16. Good, R. J.; Stromberg, R. R. ; Surface and Colloid Science, v. 11, Experimental Methods, Plenum Press, New York, 1979.

17. Marmur, A. ; Colloids Surf. A 1998, 136, 209.

18. Lander, L. M. ; Siewierski, L. M.; Brittain, W. J.; Vogler, E.; Langmuir 1993, 9, 2237.

19. Diggins D.; Fokkink, L.G.J.; Ralston, J.; Colloids Surf. 1990, 44, 299.

20. Prestidge, C. A .; Ralston, J.; J. Colloid Interface Sci. 1995, 171, 302.

21. Prestidge, C. A .; Ralston, J.; J. Colloid Interface Sci. 1996, 184, 512.

22. Debacher, N.; Ottewill, R. H.; Colloids Surf. 1991, 52, 141.

23. Vogel, A. I.; Textbook of Pratical Organic Chemistry, fifth edition, Longman Scientific and Technical, New York, 1989.

24. Debacher, N. A.; Penha, F.G.; Anais do II Congresso Brasileiro sobre Adsorção, 2000, 67.

25. Humeres, E.; Debacher, N. A.; Dias Filho, N. L.; Sierra, M. M. S.; Gonzalez, G.; J. Braz. Chem. Soc. 1992, 3, 1.

26. Fuerstenau, M.C.; Misra, M.; Palmer, B.R.; Intern. J. Miner. Process., 1990, 29, 89.

27. Fuerstenau, M.C.; Misra, M.; Palmer, B.R.; Intern. J. Miner. Process., 1990, 29, 111.

28. Buckley, A.N.; Colloids Surf. A 1994, 93, 159.

29. Gardner, J.R.; Woods, R. ; Aust. J. Chem.; 1977, 30, 981.

30. Humeres, E.; Debacher, N. A.; Wagner, T. M. ; Colloids Surf. A 1998, 149, 595. 